\title{
Overlapping Nonmatching Grids Method: Some Preliminary Studies
}

\author{
Serge Goossens, Xiao-Chuan Cai, and Dirk Roose
}

\section{Introduction}

In this paper, we report some preliminary studies of a finite difference method on overlapping nonmatching grids for a two-dimensional Poisson problem. The method can be regarded as an extension of the Generalised Additive Schwarz Method (GASM). GASM was originally developed as a preconditioning technique that uses special transmission boundary conditions at the subdomain interfaces. By involving a nonmatching grids interpolation operator in the subdomain boundary conditions, we show that the method can also be used as a discretisation scheme. We focus only on the error issues.

\section{Generalised Additive Schwarz Method}

We first recall briefly the GASM. Suppose we wish to solve $A u=f$ where $A$ represents the discretisation of a PDE defined on a domain which is partitioned into nonoverlapping subdomains. Let $R_{i}: \Omega \mapsto \Omega_{i}$ denote the linear restriction operator that maps onto subdomain $i$ by selecting the components corresponding to this subdomain. The matrix $M_{i}=R_{i} A R_{i}^{T}$ denotes the principal submatrix of the matrix $A$ associated with subdomain $\Omega_{i}$. The result of applying the GASM can be written as a sum of the solutions of independent subdomain problems, which can be solved in parallel: $M^{-1}=\sum_{i=1}^{p} R_{i}^{T} M_{i}^{-1} R_{i}$.

We describe this GASM for the case of two subdomains separated by the interface $\Gamma$. A more detailed description has been given by Tan $[\mathbf{1 2}]$ and Goossens et al. [2]. At the heart of the GASM lies an extension of the subdomains to slightly overlapping grids. With a proper definition of the overlap, the restrictions $R_{i}$ can be defined in such a way that the original discretisation is distributed across the subdomain operators $M_{i}$. Figure 1 illustrates the extension process. In case the classical five-point star stencil is used, an overlap of one mesh width is sufficient. After extension towards overlap, and thus duplication of $\Omega_{l}$ and $\Omega_{r}$ into $\Omega_{\tilde{l}}$ and $\Omega_{\tilde{r}}$ respectively, we obtain an enhanced system of equations $A u=f$ in which we still

1991 Mathematics Subject Classification. Primary 65N55; Secondary 65N22, 65F10, 65N06.

Part of this work was carried out during the visit of S. Goossens to the University of Colorado at Boulder. The financial support for this visit by the FWO-Vlaanderen is gratefully acknowledged. This research is also supported by the research Fund of K.U.Leuven (OT/94/16). S. Goossens is financed by a specialisation scholarship of the Flemish Institute for the Promotion of Scientific and Technological Research in Industry (IWT). 

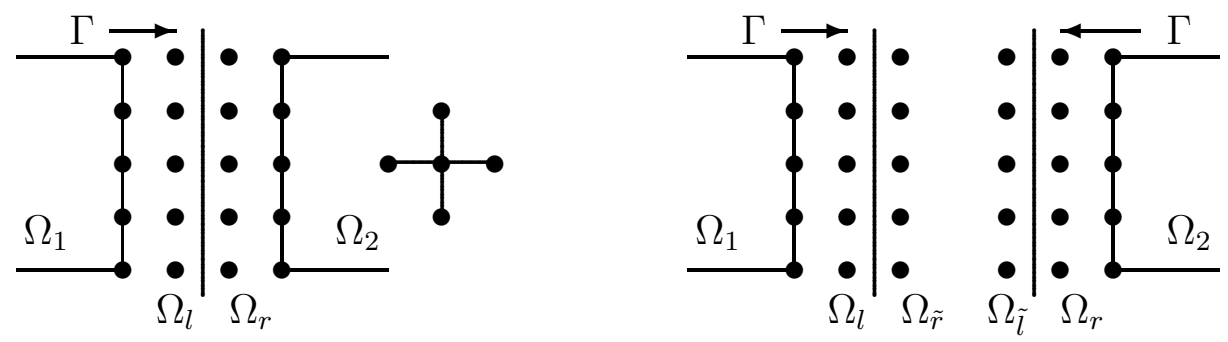

Figure 1. Extension of the subdomains to slightly overlapping grids.

have to specify the relation between the overlapping unknowns. The obvious way is just to state that the values in the duplicated subdomains $\Omega_{\tilde{l}}$ and $\Omega_{\tilde{r}}$ should be copied from the values in the original subdomains $\Omega_{l}$ and $\Omega_{r}$ respectively. This is known as the Dirichlet-Dirichlet coupling.

Tang [15] has shown that fast convergence can be obtained by choosing a good splitting, instead of increasing the overlap when a Schwarz enhanced matrix is used. Tan [12] has shown that the spectral properties of the preconditioned operator $A M^{-1}$ and thus the convergence properties of a Krylov subspace method preconditioned by a GASM, are improved by pre-multiplying the enhanced linear system $A u=f$ with a properly chosen nonsingular matrix $P$. This has been exploited by Goossens et al. [2] to accelerate the solution of the Shallow Water Equations.

This pre-multiplication with $P$ boils down to imposing more general conditions at the subdomain interfaces. This approach has originally been introduced by Lions [5] and subsequently been used by several authors. Hagstrom et. al. [3] advocate the use of nonlocal transmission conditions. Tan and Borsboom [13] have applied the Generalised Schwarz Coupling to advection-dominated problems. Nataf and Rogier $[\mathbf{8}, \mathbf{9}]$ have shown that the rate of convergence of the Schwarz algorithm is significantly higher when operators arising from the factorisation of the convection-diffusion operator are used as transmission conditions. Based on these results, Japhet [4] has developed the so-called optimised order $2(\mathrm{OO} 2)$ conditions which result in even faster convergence.

The submatrices $C_{l r}, C_{l l}, C_{r r}$ and $C_{r l}$ represent the discretisation of the transmission conditions and can be chosen freely subject to the condition that the matrix $C=\left(\begin{array}{cc}C_{l r} & -C_{l l} \\ -C_{r r} & C_{r l}\end{array}\right)$ remains nonsingular. This gives rise to the Generalised Additive Schwarz Preconditioners which are thus based on the enhanced system of equations $A u=f$ :

$$
\left(\begin{array}{cccccc}
A_{11} & A_{1 l} & 0 & 0 & 0 & 0 \\
A_{l 1} & A_{l l} & A_{l r} & 0 & 0 & 0 \\
0 & C_{l l} & C_{l r} & -C_{l l} & -C_{l r} & 0 \\
0 & -C_{r l} & -C_{r r} & C_{r l} & C_{r r} & 0 \\
0 & 0 & 0 & A_{r l} & A_{r r} & A_{r 2} \\
0 & 0 & 0 & 0 & A_{2 r} & A_{22}
\end{array}\right)\left(\begin{array}{c}
u_{1} \\
u_{l} \\
\tilde{u}_{r} \\
\tilde{u}_{l} \\
u_{r} \\
u_{2}
\end{array}\right)=\left(\begin{array}{c}
f_{1} \\
f_{l} \\
0 \\
0 \\
f_{r} \\
f_{2}
\end{array}\right) .
$$

The GASM differs from the classical Additive Schwarz Preconditioner introduced by Dryja and Widlund [1] in that the transmission conditions at the interfaces, i.e. the boundary conditions for the subdomain problems, can be changed in 

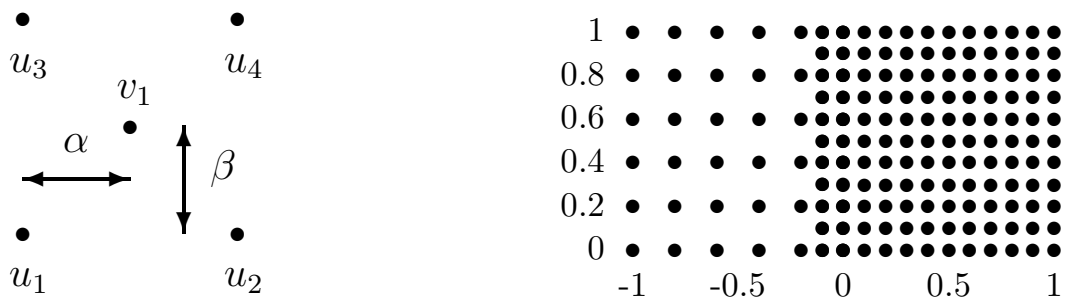

FIgURE 2. Stencil for interpolation and nonmatching grid.

order to improve the spectral properties of the preconditioned operator. An excellent description of the classical Additive Schwarz Preconditioner is given by Smith et al. [10].

\section{Nonmatching Grids}

The main topic addressed in this paper is a technique aiming at expanding the applicability of this GASM to nonmatching grids. Tan and Borsboom [14] have already shown how to apply the GASM on patched subgrids. The domain they are using, consists of a set of naturally ordered parallelograms, all of which have the same mesh width tangent to the interface. The mesh widths normal to the interface can be different on opposite sides of the interface. We want to alleviate this restriction and present a technique which also allows the GASM to be used when the mesh widths tangent to the interface are different on the opposite sides of the interface. The fact that nonmatching grids are being used implies that interpolation is necessary to transfer information from one grid to the other grid.

3.1. Consistence of grid interpolations. The following definition encapsulates an important concept in the nonmatching grids case.

DeFinition 1 (Consistent Interpolation). Let $I_{h_{j} \rightarrow h_{i}}$ be the interpolation operator from $\Omega_{j}$ to $\Omega_{i}$ with mesh parameters $h_{j}$ and $h_{i}$. Suppose $D$ is the differential operator to be approximated by a finite difference operator $D_{i}\left(L_{h_{i}}, I_{h_{j} \rightarrow h_{i}}\right)$, which depends on the usual finite difference operator $L_{h_{i}}$ and on $I_{h_{j} \rightarrow h_{i}}$. We claim that the interpolation operator $I_{h_{j} \rightarrow h_{i}}$ is consistent on $\Omega_{i}$ if

$$
\left(D-D_{i}\left(L_{h_{i}}, I_{h_{j} \rightarrow h_{i}}\right)\right) u(x)=O\left(h_{i}\right)
$$

for all $x \in \Omega_{l}$, the part of $\Omega_{i}$ that is overlapped.

The rest of this section is devoted to consistency. Bilinear interpolation is not sufficient for the interpolation operator $I_{h_{j} \rightarrow h_{i}}$. Figure 2 shows the stencil. The value of $v_{1}$ is given by $v_{1}=(1-\alpha)(1-\beta) u_{1}+\alpha(1-\beta) u_{2}+(1-\alpha) \beta u_{3}+\alpha \beta u_{4}$ where $\alpha=\left(x_{v_{1}}-x_{u_{1}}\right) /\left(x_{u_{2}}-x_{u_{1}}\right)$ and $\beta=\left(y_{v_{1}}-y_{u_{1}}\right) /\left(y_{u_{3}}-y_{u_{1}}\right)$. If in the discretisation of $-\nabla^{2} u$ in $\Omega_{l}$, the point in $\Omega_{\tilde{r}}$ does not match with a point in $\Omega_{r}$ and its value is computed by bilinear interpolation from points in $\Omega_{r}$ and $\Omega_{2}$, then this discretisation is not consistent. Hence higher order interpolation is required.

In [11], a fourth order interpolation formula was constructed with a small interpolation constant for smooth functions satisfying an elliptic equation of the form $-\left(A u_{x}\right)_{x}-\left(B u_{y}\right)_{y}+a u=f$, where $A, B>0$ and $a \geq 0$. This interpolation 
formula uses a 4 by 4 stencil. Consequently, the GASM constructed with this interpolation formula requires an extension of at least two grid lines.

Instead of using bilinear interpolation to compute $v_{1}$ from $u_{1}, u_{2}, u_{3}$ and $u_{4}$, which may result in an inconsistent discretisation, we discretise the partial differential equation on the stencil formed by $u_{1}, u_{2}, u_{3}, u_{4}$ and $v_{1}$. We seek the coefficients $\gamma_{0}, \gamma_{1}, \gamma_{2}, \gamma_{3}$ and $\gamma_{4}$ in

$$
\begin{aligned}
L(\alpha, \beta) & =\gamma_{0} u(0,0)+\gamma_{1} u((1-\alpha) h,(1-\beta) h)+\gamma_{2} u(-\alpha h,(1-\beta) h) \\
& +\gamma_{3} u(-\alpha h,-\beta h)+\gamma_{4} u((1-\alpha) h,-\beta h)
\end{aligned}
$$

so that a consistent approximation to $\left(u_{x x}+u_{y y}\right) h^{2} / 2$ at $v_{1}=u(0,0)$ is obtained. This can be done using the Taylor expansion for $u(x, y)$ about the origin: $u(x, y)=$ $u+u_{x} x+u_{y} y+u_{x x} x^{2} / 2+u_{x y} x y+u_{y y} y^{2} / 2+\mathcal{O}\left(h^{3}\right)$. We assume $|x| \leq h$ and $|y| \leq h$ so that the remainder term can be bounded by $C h^{3}$. The requirements that the coefficients of $u, u_{x}, u_{y}$ and $u_{x y}$ vanish together with the requirements that the coefficients of $u_{x x} h^{2} / 2$ and $u_{y y} h^{2} / 2$ equal 1 in the Taylor expansion of (3), yield an overdetermined system $C g=c$ :

$$
\left(\begin{array}{ccccc}
1 & 1 & 1 & 1 & 1 \\
0 & (1-\beta) & (1-\beta) & -\beta & -\beta \\
0 & (1-\alpha) & -\alpha & -\alpha & (1-\alpha) \\
0 & (1-\beta)^{2} & (1-\beta)^{2} & \beta^{2} & \beta^{2} \\
0 & (1-\alpha)^{2} & \alpha^{2} & \alpha^{2} & (1-\alpha)^{2} \\
0 & (1-\alpha)(1-\beta) & -\alpha(1-\beta) & \alpha \beta & -(1-\alpha) \beta
\end{array}\right)\left(\begin{array}{l}
\gamma_{0} \\
\gamma_{1} \\
\gamma_{2} \\
\gamma_{3} \\
\gamma_{4}
\end{array}\right)=\left(\begin{array}{l}
0 \\
0 \\
0 \\
1 \\
1 \\
0
\end{array}\right)
$$

This overdetermined system $C g=c$ can only have solutions if the determinant of the enhanced matrix $\tilde{C}=(C \mid c)$ is zero:

$$
\operatorname{det} \tilde{C}=\operatorname{det}(C \mid c)=(\beta-\alpha)(\alpha+\beta-1) .
$$

Hence a solution can only exist when $\alpha=\beta$ or $\alpha+\beta=1$, i.e. when the point $v_{1}$ lies on one of the diagonals of the square, formed by $u_{1}, u_{2}, u_{3}$ and $u_{4}$. The solution is

$$
g=\left(\begin{array}{lllll}
-2-(1-\alpha) / \alpha-\alpha /(1-\alpha) & \alpha /(1-\alpha) & 1 & (1-\alpha) / \alpha & 1
\end{array}\right)^{T}
$$

when $\alpha=\beta$ and when $\alpha+\beta=1$ it is

$$
g=(-2-(1-\alpha) / \alpha-\alpha /(1-\alpha) \quad 1 \quad(1-\alpha) / \alpha \quad 1 \quad \alpha /(1-\alpha))^{T} .
$$

The truncation error is determined by substitution of this solution in (3):

$$
L(\alpha, \beta)=h^{2}\left(u_{x x}+u_{y y}\right) / 2+C_{\alpha} h^{3}\left(u_{x x x}+u_{y y y}\right) / 6+\mathcal{O}\left(h^{4}\right),
$$

where $C_{\alpha}=1-2 \alpha$ in case $\alpha=\beta$ and $C_{\alpha}=2 \alpha-1$ when $\alpha+\beta=1$. Hence an $\mathcal{O}(h)$ approximation to $u_{x x}+u_{y y}$ can be obtained, an $\mathcal{O}\left(h^{2}\right)$ approximation can only be obtained when $\alpha=\beta=1 / 2$ :

$$
L(\alpha, \beta)=h^{2}\left(u_{x x}+u_{y y}\right) / 2+h^{4}\left(u_{x x x x}+6 u_{x x y y}+u_{y y y y}\right) / 96+\mathcal{O}\left(h^{6}\right) .
$$

In summary, a consistent discretisation exists only if $v_{1}$ is in the center or on one of the diagonals of the square formed by $u_{1}, u_{2}, u_{3}$ and $u_{4}$. The truncation error is $O\left(h^{2}\right)$ when $v_{1}$ is in the center and is $O(h)$ when $v_{1}$ is on one of the diagonals. 
3.2. Error Analysis. Miller [6] has proven the convergence of the Schwarz algorithm based on a maximum principle. We restrict ourselves here to showing that second order accuracy in the $L_{\infty}$ norm is obtained when a consistent interpolation is used. The convergence of the GASM will be studied elsewhere.

We denote by $p=(i, j)$ an index pair. $J_{\Omega}$ is the set of index pairs of grid points in the domain $\Omega$. We make the following assumptions.

1. For all $p \in J_{\Omega}: \mathcal{L}_{h}$ has the form: $\mathcal{L}_{h} u_{p}=-c_{p} u_{p}+\sum_{k} c_{k} u_{k}$ where the coefficients are positive and the sum over $k$ is taken over mesh points which are neighbours of $p$.

2. For all $p \in J_{\Omega}: c_{p} \geq \sum_{k} c_{k}$.

3. The set $J_{\Omega}$ is connected. By definition a point is connected to each of its neighbours occurring in (1) with a nonzero coefficient. By definition a set is connected if, given any two points $p$ and $q$ in $J_{\Omega}$, there is a sequence of points $p=p_{0}, p_{1}, \ldots, p_{m+1}=q$, such that each point $p_{i}$ is connected to $p_{i-1}$ and $p_{i+1}$, for $i=1,2, \ldots, m$.

4. At least one of the equations must involve a Dirichlet boundary condition.

The maximum principle as given by Morton and Meyers [7] can briefly be stated as follows.

Lemma 2 (Maximum Principle [7]). Suppose that $\mathcal{L}_{h}, J_{\Omega}$ and $J_{\partial \Omega}$ satisfy all the assumptions mentioned above and that a mesh function $u_{p}$ satisfies $\mathcal{L}_{h} u_{p} \geq 0$ for all $p \in J_{\Omega}$. Then $u_{p}$ cannot attain a nonnegative maximum at an interior point:

$$
\max _{p \in J_{\Omega}} u_{p} \leq \max \left\{\max _{a \in J_{\partial \Omega}} u_{a}, 0\right\} .
$$

THEOREM 3. Suppose a nonnegative mesh function $\Phi_{p}$ is defined on $J_{\Omega} \cup J_{\partial \Omega}$ such that $\mathcal{L}_{h} \Phi_{p} \geq 1$ for all $p \in J_{\Omega}$ and that all the assumptions mentioned above are satisfied. Then the error in the approximation is bounded by

$$
\left|e_{p}\right| \leq \max _{a \in J_{\partial \Omega}} \Phi_{a} \max _{p \in J_{\Omega}}\left|T_{p}\right|
$$

where $T_{p}$ is the truncation error.

To prove second order accuracy in the $L_{\infty}$ norm, we show that the discretisation of $\nabla^{2}(-u)=f$ and the coupling equations satisfy the assumptions (1) and (2) for the maximum principle. The comparison function is chosen as $\Phi(x, y)=$ $\left((x-\mu)^{2}+(y-\nu)^{2}\right) / 4$, resulting in $\mathcal{L}_{h} \Phi_{p}=1$ for all $p \in J_{\Omega}$. The scalars $\mu$ and $\nu$ are chosen to minimise the maximum value of this function $\Phi(x, y)$ on the boundary $\partial \Omega$. The classical five-point discretisation of $\nabla^{2} u$

$$
\mathcal{L}_{h_{i}} u_{p}=\left(u_{i-1, j}+u_{i, j-1}-4 u_{i, j}+u_{i+1, j}+u_{i, j+1}\right) / h_{i}^{2}
$$

satisfies the assumptions for the maximum principle. In case (9) is used to obtain an equation for $v_{1}$, the assumptions (1) and (2) for the maximum principle are satisfied since this equation has $c_{k}=1 /\left(2 h_{i}^{2}\right)$ and $c_{p}=4 /\left(2 h_{i}^{2}\right)$. For problems with at least one Dirichlet boundary condition, the standard error analysis using the maximum principle yields second order accuracy in the $L_{\infty}$ norm. The proof is essentially the same as the one given by Morton and Meyers [7].

If the point $v_{1}$ is not in the center of the square formed by $u_{1}, u_{2}, u_{3}$ and $u_{4}$, we have to use (8) to obtain an equation from which $v_{1}$ can be determined. In this case we still have second order accuracy, but a different comparison function must be defined in $\Omega_{l}$. This is analogous to the classical result that second order accuracy is obtained with a second order discretisation of the partial differential equation and only first order discretisation of the boundary conditions. 
TABLE 1. Results for $u_{1}(x, y)=\exp \left(-x^{2}-y^{2}\right)$.

\begin{tabular}{|c|c|c|c|c|c|}
\hline \multicolumn{6}{|c|}{ Results for $h_{0}=2 h_{1}$. } \\
\hline$n_{0}$ & $n_{1}$ & $L_{\infty}$ in block 0 & ratio & $L_{\infty}$ in block 1 & ratio \\
\hline 6 & 11 & 0.00173386 & & 0.00160317 & \\
\hline 11 & 21 & 0.000492551 & 3.52016 & 0.000439111 & 3.65094 \\
\hline 21 & 41 & 0.000128595 & 3.83025 & 0.000113528 & 3.86787 \\
\hline 41 & 81 & $3.28033 \mathrm{e}-05$ & 3.92018 & $2.87286 \mathrm{e}-05$ & 3.95174 \\
\hline 81 & 161 & $8.28181 \mathrm{e}-06$ & 3.96089 & $7.21997 \mathrm{e}-06$ & 3.97905 \\
\hline$n_{0}$ & $n_{1}$ & $L_{2}$ in block 0 & ratio & $L_{2}$ in block 1 & ratio \\
\hline 6 & 11 & 0.0006622 & & 0.00051287 & \\
\hline 11 & 21 & 0.000194007 & 3.41328 & 0.000137398 & 3.73273 \\
\hline 21 & 41 & $5.20312 \mathrm{e}-05$ & 3.72867 & $3.51015 \mathrm{e}-05$ & 3.91431 \\
\hline 41 & 81 & $1.34408 \mathrm{e}-05$ & 3.87114 & $8.8388 \mathrm{e}-06$ & 3.9713 \\
\hline 81 & 161 & $3.41357 \mathrm{e}-06$ & 3.93746 & $2.2156 \mathrm{e}-06$ & 3.98935 \\
\hline \multicolumn{6}{|c|}{ Reference results for $h_{0}=h_{1}$. } \\
\hline$n_{0}$ & $n_{1}$ & $L_{\infty}$ in block 0 & $L_{2}$ in block 0 & $L_{\infty}$ in block 1 & $L_{2}$ in block 1 \\
\hline 6 & 6 & 0.00288425 & 0.0010893 & 0.00288425 & 0.00117384 \\
\hline 11 & 11 & 0.000736578 & 0.000280968 & 0.000736578 & 0.000299917 \\
\hline 21 & 21 & 0.000185341 & $7.12195 \mathrm{e}-05$ & 0.000185341 & $7.41716 \mathrm{e}-05$ \\
\hline 41 & 41 & $4.63929 \mathrm{e}-05$ & $1.79235 \mathrm{e}-05$ & $4.63929 \mathrm{e}-05$ & $1.83303 \mathrm{e}-05$ \\
\hline 81 & 81 & $1.16036 \mathrm{e}-05$ & $4.49551 \mathrm{e}-06$ & $1.16036 \mathrm{e}-05$ & $4.54876 \mathrm{e}-06$ \\
\hline 161 & 161 & $2.90103 \mathrm{e}-06$ & $1.1257 \mathrm{e}-06$ & $2.90103 \mathrm{e}-06$ & $1.1325 \mathrm{e}-06$ \\
\hline
\end{tabular}

\section{Numerical Examples}

The testcases are concerned with the solution of

$$
-\nabla^{2} u=f \text { on } \Omega \text { and } u=g \text { on } \partial \Omega \text {. }
$$

The domain $\Omega=\Omega_{0} \cup \Omega_{1}$ consists of two subdomains $\Omega_{0}=(-1,0) \times(0,1)$ and $\Omega_{1}=\left(-h_{1}, 1\right) \times(0,1)$. The coordinates of the grid points are $\left(x_{i}, y_{j}\right)$, where $x_{i}=x_{\text {ref }}+i h$ and $y_{j}=y_{\text {ref }}+j h$, for $i=0,1, \ldots,\left(n_{0}-1\right)$ for block $0 ; i=0,1, \ldots, n_{1}$ for block 1 and $j=0,1, \ldots,(n-1)$. The reference point for block 0 is $(-1,0)$ and for block 1 it is $\left(-h_{1}, 0\right)$. The grid sizes are $h_{0}=1 /\left(n_{0}-1\right)$ and $h_{1}=1 /\left(n_{1}-1\right)$. The interface $\Gamma$ is defined by $x=-h_{1} / 2$. The right-hand side $f$ and the boundary conditions $g$ are chosen such that the exact solution is $u_{1}$ resp. $u_{2}$ in the testcases, where $u_{1}(x, y)=\exp \left(-x^{2}-y^{2}\right)$ and $u_{2}(x, y)=\exp (\alpha x) \sin (\beta y)$, where $\alpha=2$ and $\beta=8 \pi$.

By definition the error is $e_{i, j}=u\left(x_{i}, y_{j}\right)-u_{i, j}$ where $u\left(x_{i}, y_{j}\right)$ is the exact solution and $u_{i, j}$ is the computed approximation. In Tables 1-3 we list both the $L_{\infty}$ norm and $L_{2}$ norm of the error, defined by $L_{\infty}(e)=\max _{i, j}\left|e_{i, j}\right|$ and $L_{2}(e)=$ $\sqrt{\frac{1}{n^{2}} \sum_{i=0}^{n-1} \sum_{j=0}^{n-1} e_{i, j}^{2}}$.

The results for $-\nabla^{2} u_{1}=f_{1}$ on a nonmatching grid are given in Table 1 . We also give the results for the same problem on matching grids. This allows us to verify the accuracy of the results. In Table 2 we give the results for $-\nabla^{2} u_{2}=f_{2}$. The ratios in the fourth and sixth columns approach 4 as the mesh widths are divided by 2 , showing that the method is second order accurate.

To emphasize the importance of consistent interpolation, we give in Table 3 the results for $-\nabla^{2} u_{2}=f_{2}$ when bilinear interpolation is used. In this case (2) is not 
TABLE 2. Results for $u_{2}(x, y)=\exp (2 x) \sin (8 \pi y)$.

\begin{tabular}{|cc|cc|cc|}
\hline \multicolumn{5}{|c|}{ Results for $h_{0}=2 h_{1}}$. \\
\hline$n_{0}$ & $n_{1}$ & $L_{\infty}$ in block 0 & ratio & $L_{\infty}$ in block 1 & ratio \\
\hline 6 & 11 & 7.23983 & & 4.21601 & \\
11 & 21 & 0.48669 & 14.8756 & 0.718661 & 5.86648 \\
21 & 41 & 0.102748 & 4.73673 & 0.17356 & 4.14071 \\
41 & 81 & 0.0249472 & 4.11862 & 0.0453624 & 3.82608 \\
81 & 161 & 0.00656458 & 3.80027 & 0.011314 & 4.0094 \\
\hline \hline$n_{0}$ & $n_{1}$ & $L_{2}$ in block 0 & ratio & $L_{2}$ in block 1 & ratio \\
\hline 6 & 11 & 3.20061 & & 1.66157 & \\
11 & 21 & 0.204226 & 15.6719 & 0.303296 & 5.47838 \\
21 & 41 & 0.0435961 & 4.6845 & 0.0748621 & 4.0514 \\
41 & 81 & 0.01074 & 4.05923 & 0.0188233 & 3.9771 \\
81 & 161 & 0.00269816 & 3.98049 & 0.00473253 & 3.97743 \\
\hline \hline \multicolumn{7}{c}{ Reference results for $h_{0}=h_{1}}$. \\
\hline$n_{0}$ & $n_{1}$ & $L_{\infty}$ in block 0 & $L_{2}$ in block 0 & $L_{\infty}$ in block 1 & $L_{2}$ in block 1 \\
\hline 6 & 6 & 18.0965 & 6.69002 & 47.1923 & 21.3855 \\
11 & 11 & 0.717338 & 0.264132 & 3.3735 & 1.40949 \\
21 & 21 & 0.136687 & 0.0502781 & 0.718661 & 0.301632 \\
41 & 41 & 0.0321275 & 0.011811 & 0.17356 & 0.074284 \\
81 & 81 & 0.00831887 & 0.00290772 & 0.0453624 & 0.0186726 \\
161 & 161 & 0.00207194 & 0.000724103 & 0.011314 & 0.00469548 \\
\hline \multicolumn{7}{c}{} \\
\hline
\end{tabular}

TABLE 3. Results for $u_{2}(x, y)=\exp (2 x) \sin (8 \pi y)$ when bilinear interpolation is used.

\begin{tabular}{|cc|cc|cc|}
\hline$n_{0}$ & $n_{1}$ & $L_{\infty}$ in block 0 & $L_{\infty}$ in block 1 & $L_{2}$ in block 0 & $L_{2}$ in block 1 \\
\hline 7 & 12 & 9.63784 & 7.65477 & 4.32959 & 2.7709 \\
12 & 22 & 0.847174 & 0.995454 & 0.262247 & 0.366744 \\
22 & 42 & 0.142845 & 0.220307 & 0.048968 & 0.082186 \\
42 & 82 & 0.0315985 & 0.0496966 & 0.011165 & 0.0197278 \\
82 & 162 & 0.00710074 & 0.0118877 & 0.00271561 & 0.00485607 \\
\hline
\end{tabular}

satisfied. The results are for the same problem but solved on shifted grids. The reason for using shifted grids is that an interpolation is required for every point in $\Omega_{\tilde{r}}$, while for grids as in Fig. 2 only half of the points in $\Omega_{\tilde{r}}$ require an interpolation since the other points match some point in $\Omega_{r}$. The coordinates of the grid points are now given by $\left(x_{i}, y_{j}\right)$ where $x_{i}=x_{\text {ref }}+\left(i-\frac{1}{2}\right) h$ and $y_{j}=y_{\text {ref }}+\left(j-\frac{1}{2}\right) h$ for $i=0,1, \ldots,(n-1)$ and $j=0,1, \ldots,(n-1)$. The reference point for block 0 is $(-1,0)$ and for block 1 it is $(0,0)$. The grid sizes $h_{0}=1 /\left(n_{0}-2\right)$ and $h_{1}=1 /\left(n_{1}-2\right)$ are the same as in the previous case. Since an inconsistent interpolation is used, the error is larger.

\section{Concluding Remarks}

We studied an overlapping nonmatching grids finite difference method. A consistency condition is introduced for the nonmatching grids interpolation operator, and under the consistency condition we proved second order global accuracy of the discretisation scheme. 


\section{References}

1. M. Dryja and O. B. Widlund, An additive variant of the Schwarz alternating method for the case of many subregions, Tech. Report 339, Department of Computer Science, Courant Institute, 1987.

2. S. Goossens, K. Tan, and D. Roose, An efficient FGMRES solver for the shallow water equations based on domain decomposition, Proc. Ninth Int. Conf. on Domain Decomposition Meths. (P. Bjørstad, M. Espedal, and D. Keyes, eds.), 1996.

3. T. Hagstrom, R. P. Tewarson, and A. Jazcilevich, Numerical experiments on a domain decomposition algorithm for nonlinear elliptic boundary value problems, Appl. Math. Lett. 1 (1988), no. 3, 299-302.

4. C. Japhet, Optimized Krylov-Ventcell method. Application to convection-diffusion problems, Proc. Ninth Int. Conf. on Domain Decomposition Meths. (P. Bjørstad, M. Espedal, and D. Keyes, eds.), 1996.

5. P. L. Lions, On the Schwarz alternating method III: A variant for nonoverlapping subdomains, Proc. Third Int. Conf. on Domain Decomposition Meths. (Philadelphia) (T. F. Chan, R. Glowinski, J. Périaux, and O. B. Widlund, eds.), SIAM, 1990, pp. 202-223.

6. K. Miller, Numerical analogs to the Schwarz alternating procedure, Numer. Math. 7 (1965), 91-103.

7. K. W. Morton and D. F. Mayers, Numerical solution of partial differential equations, Cambridge University Press, 1994.

8. F. Nataf and F. Rogier, Factorization of the convection-diffusion operator and the Schwarz algorithm, Mathematical Models and Methods in Applied Sciences 5 (1995), no. 1, 67-93.

9. __ Outflow boundary conditions and domain decomposition method, Domain Decomposition Methods in Scientific and Engineering Computing (Providence) (D. E. Keyes and J. Xu, eds.), Contemporary Mathematics, no. 180, AMS, 1995, pp. 289-293.

10. B. F. Smith, P. E. Bjørstad, and W. D. Gropp, Domain decompostion: Parallel multilevel methods for elliptic partial differential equations, Cambridge University Press, 1996.

11. G. Starius, Composite mesh difference methods for elliptic boundary value problems, Numer. Math. 28 (1977), 243-258.

12. K. H. Tan, Local coupling in domain decomposition, Ph.D. thesis, Universiteit Utrecht, 1995.

13. K. H. Tan and M. J. A. Borsboom, On generalized Schwarz coupling applied to advectiondominated problems, Domain Decomposition Methods in Scientific and Engineering Computing (Providence) (D. E. Keyes and J. Xu, eds.), Contemporary Mathematics, no. 180, AMS, 1995, pp. 125-130.

14. - Domain decomposition with patched subgrids, Domain Decomposition Methods in Sciences and Engineering (Chichester) (R. Glowinski, J. Periaux, Z-C. Shi, and O. Widlund, eds.), John Wiley \& Sons Ltd., 1997, pp. 117-124.

15. W. P. Tang, Generalized Schwarz Splittings, SIAM J. Sci. Stat. Comput. 13 (1992), no. 2, $573-595$.

Department of Computer Science, Katholieke Universiteit Leuven, CelestijnenlaAn 200A, B-3001 Heverlee, Belgium

E-mail address: Serge.Goossens@cs.kuleuven.ac.be

Department of Computer Science, University of Colorado at Boulder, Campus Box

430, Boulder, Colorado 80309-0430, USA

E-mail address: cai@cs.colorado.edu

Department of Computer Science, Katholieke Universiteit Leuven, CelestijnenlaAn 200A, B-3001 Heverlee, Belgium

E-mail address: Dirk.Roose@cs.kuleuven.ac.be 